Egyptian Journal of Aquatic Biology \& Fisheries

Zoology Department, Faculty of Science,

Ain Shams University, Cairo, Egypt.

ISSN $1110-6131$

Vol. 24(5): $261-277(2020)$

www.ejabf.journals.ekb.eg

\title{
Effect of heavy metals in irrigation water of different fish farms on the quality of cultured fish
}

\section{Zakaria I. M. El-Khatib ${ }^{1}$; Ahmad M. Azab ${ }^{1 *}$; Hamdy A. H. Abo-Taleb ${ }^{1}$; Ahmed N.M. Al-Absawy ${ }^{1}$ and Mohamed M.M. Toto ${ }^{2}$}

1. Marine Biology and Ichthyology Section, Zool. Dept., Fac. of Sci., Al-Azhar University, Cairo, Egypt

2. Fish nutrition, National Institute of Oceanography and Fisheries (NIOF)

*Corresponding author: Ahmadmosaad.201@azhar.edu.eg

\section{ARTICLE INFO}

Article History:

Received: July 3, 2020

Accepted: July 23, 2020

Online: July 25,2020

Keywords:

Nile tilapia;

Oreochromis niloticus;

Heavy metals;

Egypt,

Fish farm;

Contamination Factor;

Pollution load Index;

Metal Pollution Index

\section{ABSTRACT}

The present study aimed to study the effect of irrigation water quality in different fish farms in Egypt on the quality of Nile tilapia, Oreochromis niloticus, produced from these fish farms. Four fish farms (El-Abbasa, Edko, Mariout and Bahr El-Baqar) which irrigated with different water sources were selected to study the effect of some heavy metals (iron, copper, zinc and lead) on fish quality produced from these farms during the period of fish culturing from April 2018 to November 2018.

Results indicated that most of the highest averages of all heavy metal concentrations in water, sediment and fish muscular tissues were recorded from Bahr El-Baqar fish farm and their lowest values from El-Abbasa fish farm. The highest contamination factors for most heavy metals were recorded in Bahr El-Baqar fish farm and the lowest in El-Abbasa fish farm. The present study concluded that El-Abbassa fish farm has low pollution and the other fish farms (Edko, Mariout, and Bahr El-Baqar) have progressive deterioration of the site quality.

\section{INTRODUCTION}

Fish is an important economic source of protein compared to other sources of animal protein. In developing countries, fish contribute about $30 \%$ of the total consumption of animal protein per capita (Wang et al., 2015). Aquaculture contributes more than half of the total fish production in the world (Subasinghe et al., 2009). Tilapia inhabits a variety of fresh water habitats including shallow streams, ponds, rivers and lakes. Historically they have been of major importance in artisan fishing in Africa and are of increasing importance in aquaculture (Afami \& Okories, 2008).

The pollution of the aquatic environment with heavy metals has become a worldwide problem, because they are indestructible and most of them have toxic effects on organisms (MacFarlane \& Burchett, 2000). Pollution of the aquatic environment by inorganic chemicals has been considered a major threat to the aquatic organisms including fishes. The agricultural drainage water containing pesticides and fertilizers and effluents of industrial activities and 
runoffs in addition to sewage effluents supply the water bodies and sediment with huge quantities of inorganic anions and heavy metals (ECDG, 2002). Toxic and harmful pollutants of aquatic environment pose a very serious danger to human health and his life because they can bioaccumulate in fish tissues and they cannot be destroyed or degraded through biological degradation (Al-Kenawy \& Aly, 2015).

Metals can be incorporated into food chains and concentrated in aquatic organisms to levels that affect their physiological state of the effective pollutants are the heavy metals which have drastic environmental impact on all organisms. Trace metals such as iron, copper, lead and zinc play a biochemical role in the life processes of all aquatic plants and animals; therefore, they are essential in the aquatic environment in trace amounts (Mason, 2002). But accumulation of heavy metals in large amount in aquatic organisms can pose a long-lasting effect on biogeochemical cycling in the ecosphere. Heavy metals can also adversely affect the growth rate of fish. The presence of heavy metals in different foods constitutes a serious health hazards depending on their relative levels (Nussey, 1998; Mansour \& Sidky 2002; Momtaz, 2002; ElEnany, 2004; Duruibe et al., 2007; Al-Haidarey, 2009; Abdel-Rasheed, 2011and Balasim, 2013)

In Egypt, Environmental pollution with heavy metals is one of the biggest problems that face human being (Al-Naggar $\boldsymbol{e t} \boldsymbol{a l}, \mathbf{2 0 1 8}$ ). Bahr El-Baqar drain is considered as one of the most polluted drains in Egypt (Abdel-Shafy \& Aly, 2002). The total discharge pumped to Lake Manzala from that drain is $2.3 \mathrm{BCM} /$ year. It is passing through four highly populated Governorates; Qalubeya, Sharkia, Ismailia and Port Said. Unfortunately, at the last decades, great areas on both sides of the drain were using its polluted water for irrigation and fish culture (Hamed, 2013). Lake Mariout is one of the main fishing grounds of Alexandria (Saad et al., 2017). Lake Mariout was considered as an oxidation pond for Alexandrian waste water and agricultural wastes, discharged to it by the newly constructed plant stations (El-Zokm et al., 2018).

Thus, the present study aimed to study the effect of irrigation water quality in different fish farms in Egypt on the quality of Nile tilapia, Oreochromis niloticus, produced from these fish farms.

\section{MATERIALS AND METHODS}

\section{A. Study areas:}

This study was investigated in four fish farms irrigated with different water sources, the location of each fish farm was presented in Table (1).

1-El-Abbasa fish farm: one of the most productive Egyptian fish farms, it is located in ELSharkia Governorate. The fish farm is about 2200 feddans (Table 1).

2- Edko fish farm: Lake Edko with a depth ranged from 50 to $180 \mathrm{~cm}$. reached about 700 $\mathrm{km}^{2}$. It receives its water from two sources. The main source is the drainage water of Kom Belag and Bersik drains $(1836.55 \times 106 \mathrm{~m} 3)$. The sea water of Abu Qir Bay enters the lake sometimes through the lake sea connection as subsurface water current under the action of wind especially in winter.

3- Mariout fish farm: It is at the western extremity of Alexandria city, in the vicinity of Lake Mariout and about $1 \mathrm{Km}$ south of the Mediterranean Sea coast. This farm is affected by ElMex Pumping Station that pumps out the water through El-Umum drain which connects the Lake Mariout to the Mediterranean Sea through by a channel of about $800 \mathrm{~m}$ long. 
4- Bahr El-Baqar fish farm: Bahr El-Baqar drain system consists of a main drain that starts near the city of Zagazig where it collects the effluents from two secondary drains.

Table (1): Location of the selected fish farms

\begin{tabular}{|l|l|}
\hline \multicolumn{1}{|c|}{ Fish farms } & \multicolumn{1}{c|}{ Location } \\
\hline El-Abbassa fish farm & $30^{\circ} 32^{\prime}$ N. $31^{\circ} 44^{\prime} \mathrm{E}$. \\
\hline Edko fish farm & $31^{\circ} 13^{\prime} \mathrm{N} .30^{\circ} 17^{\prime} \mathrm{E}$. \\
\hline Mariout fish farm & $31^{\circ} 145^{\prime}$ N. $29^{\circ} 852^{\prime} \mathrm{E}$. \\
\hline Bahr El-Baqar fish farm & $30^{\circ} 58^{\prime} \mathrm{N} .32^{\circ} 08 \mathrm{E}$ \\
\hline
\end{tabular}

\section{B- Fish sampling:}

A total of 324 specimens of Oreochromis niloticus (Figure 1) were monthly collected from different fish farms. 81 specimens of El-Abbassa fish farm (8.3-25.9 cm in total length and 20.1-320.7 $\mathrm{g}$ in weight); 81 specimens of Edko fish farm (7.8-25.7 cm TL and $20.50-333.7 \mathrm{~g}$ in $\mathrm{W}) ; 81$ specimens of Mariout fish farm (11.70 -22.80 cm TL and $25.50-304.5 \mathrm{~g} \mathrm{~W})$, and 81 specimens of Bahr El-Baqar fish farm $(8.40$ - $25.70 \mathrm{~cm}$ TL and $20.00-280.40 \mathrm{~g} \mathrm{~W})$ during the period from April, 2018 to November, 2018.

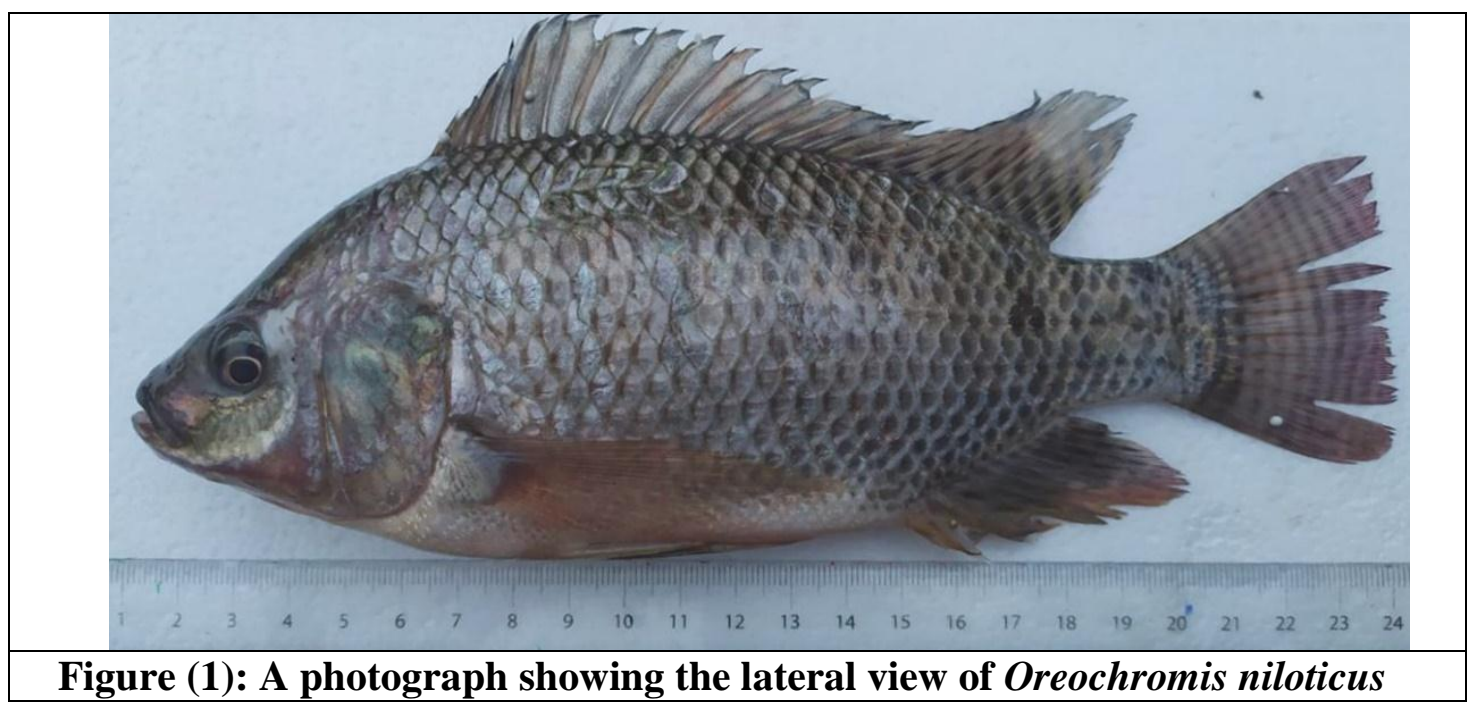

\section{C- Determination of heavy metals in water samples:}

Surface water samples at four fish farms were seasonally taken by a vertical PVC water sampler at depth of a half meter from the water surface. Samples of 1 liter were placed in polyethylene bottles and transferred to the laboratory for heavy metal analysis. All water samples were collected from each fish farm in triplicate and each sample was measured also in triplicate.

Heavy metals considered here were; $\mathrm{Fe}, \mathrm{Cu}, \mathrm{Zn}$ and $\mathrm{Pb}$. Concentrations of heavy metals in the water were determined after the digestion by nitric acid according to APHA (2005). Heavy metals were measured by atomic absorption spectrophotometer (model; Perkin Elmer, 3150) was used for measuring the optical density for each element at its corresponding wave length (Fe 248.3, $\mathrm{Cu} 324.8, \mathrm{Zn} 213.9$ and $\mathrm{Pb} 217 \mathrm{~nm})$. Results were expressed as $\mu \mathrm{g} / \mathrm{L}$. 
The concentrations of heavy metals were measured according to the following equation:

Where:

\section{Metal concentration $(\mu \mathrm{g} / \mathrm{L})=\mathrm{A} \times \mathrm{B} / \mathrm{C}$}

$\mathbf{A}=$ Conc. of metal in digested solution $\mu \mathrm{g} / \mathrm{L}$.

$\mathbf{B}=$ Final volume of digested solution, $\mathrm{ml}$.

$\mathbf{C}=$ Sample volume, $\mathrm{ml}$.

\section{D- Determination of heavy metals in sediment samples:}

Sediment samples were collected seasonally from the bottom of each fish farm using Ekman dredge bottom sampler at the same time of water collection. Then, these samples were transferred into the laboratory in ice box. The preparation and complete digestion of the sediment samples were done according to Martin \& Martin (1989) and David \& Janice (1989).

Heavy metals ( $\mathrm{Fe}, \mathrm{Cu}, \mathrm{Zn}$, and $\mathrm{Pb}$ ) were determined by atomic absorption spectrometry at corresponding wave lengths according to the following equation:

Metal conc. $(\mu \mathrm{g} / \mathrm{g}$ dry wt. $)=$ Conc. of metal in digest solution $(\mu \mathrm{g} / \mathrm{L}) \times$ its vol. $(\mathrm{ml})$.

\section{E- Determination of heavy metals in fish tissue:}

Fish samples were stored in prewashed polyethylene bags and transferred immediately to the laboratory in ice box at $4^{\circ} \mathrm{C}$. In the laboratory, fishes were identified and all morphological characters for each sample were recorded. Each fish was dissected and its muscle tissue $(0.5$ g) was cut and placed in Teflon vessel and $5 \mathrm{ml}$ of nitric acid (ultrapure) was added to soft tissue. The vessels were tightly covered and allowed to predigest at room temperature overnight. Samples were digested on a hot plate at $100{ }^{\circ} \mathrm{C}$ for $2 \mathrm{hrs}$. Then they cooled at room temperature. If the solution was not clear, it was reheated for another $1 \mathrm{hr}$. at $100{ }^{\circ} \mathrm{C}$. The samples were transferred to $25 \mathrm{ml}$ volumetric flasks. Before analysis, samples were filtered. All samples were analyzed three times for $\mathrm{Fe}, \mathrm{Cu}, \mathrm{Zn}$ and $\mathrm{Pb}$, using flame atomic absorption spectrometry (model Varian AA240FS) according to the corresponding wave lengths (UNEP, 1984). Concentrations were expressed in terms of wet weight as microgram per gram according to the following equation:

Metal conc. $(\mu \mathrm{g} / \mathrm{g}$ wet wt. $)=$ Conc. of metal in digest solution $(\mu \mathrm{g} / \mathrm{l}) \mathrm{x}$ its vol. $(\mathrm{ml})$

In fish from the aquatic ecosystem, which includes water and sediments, the bioconcentration factor (BAF) and bio-sedimentation factor (BSF) are calculated according to the following:

$$
\mathrm{BAF}=\mathrm{M}_{\text {tissue }}(\mathrm{mg} / \mathrm{kg}) / \mathrm{M}_{\text {water }}(\mathrm{mg} / \mathrm{l})
$$

(Orata \& Birgen, 2016)

Where: $\mathbf{M}_{\text {tissue: }}$ is the metal concentration in fish's tissues

$\mathbf{M}_{\text {water: }}$ is the metal concentration in water.

$$
\mathrm{BSF}=\mathrm{M}_{\text {tissue }}(\mathrm{mg} / \mathrm{kg}) / M_{\text {sediment }}(\mathrm{mg} / \mathrm{kg})
$$

(Evans \& Engel (1994)

Where: $\mathbf{M}_{\text {tissue }}$ : is the metal concentration in fish's tissues

$\mathbf{M}_{\text {sediment }}$ : is the metal concentration in sediment. 


\section{F. Heavy Metal Contamination Indices:}

Heavy metal contamination indices could be evaluated by the determination of the contamination factor (CF), pollution load index (PLI), and metal pollution index (MPI) from the following formulae.

$$
\mathbf{C F}=\mathrm{C}_{\text {metal }} / \mathrm{C}_{\text {background }}
$$

\section{(Harikumar \& Jisha 2010)}

Where: $\mathbf{C}_{\text {metal }}=$ mean metal conc . in water or sediment

$\mathbf{C}_{\text {background }}=$ background conc. of that metal

Contamination factor $(\mathrm{CF})$ referred that $\mathrm{CF}<1$ refers to low contamination, $1 \geq \mathrm{CF}<3$ means moderate contamination, $3 \geq \mathrm{CF} \geq 6$ indicates considerable contamination, and $\mathrm{CF}>6$ indicates very high contamination (Harikumar \& Jisha 2010).

$$
\mathrm{PLI}=\left(\mathrm{CF}_{1} \times \mathrm{CF}_{2} \times \mathrm{CF}_{3} \times \ldots . . . . \mathrm{CF}_{n}\right)^{1 / n}
$$

(Tomlinson et al,. 1980)

Where: $\mathbf{n}=$ number of the studied metals

\section{$\mathbf{C F}=$ Contamination factor for water or sediment}

When the value of PLI is < 1, a low of pollution is suggested. The PLI value of one indicates the presence of only baseline level of pollutants while value above one indicates progressive deterioration of the site and estuarine quality.

$$
\text { MPI }=\left(M_{1} \times M_{2} \times M_{3} \times \cdots \cdots \times M_{n}\right)^{1 / n} \quad \text { (Caeiro et al., 2005) }
$$

Where: $\mathbf{n}=$ number of the studied metals

$\mathbf{M}=$ metal concentration

Permissible levels reported by FAO (1992) were 1, 0.05, 5, 0.3 and 0.01 (ppm) for water $\mathrm{Cu}, \mathrm{Pb}, \mathrm{Zn}, \mathrm{Fe}$ and $\mathrm{Cd}$ respectively.

\section{RESULTS}

\section{1- Heavy metal concentration $(\mu \mathrm{g} / \mathrm{l})$ in the water:}

\section{1- Iron concentration:}

Results showed that the highest average value of iron concentration in water was recorded at Bahr El-Baqar fish farm $(863.21 \pm 67.07 \mu \mathrm{g} / \mathrm{l})$ and the lowest value at El-Abbassa fish farm $(116.59 \pm 16.94 \mu \mathrm{g} / \mathrm{l})$. The highest concentration of iron was recorded during spring $(1043.94 \pm 58.83 \mu \mathrm{g} / \mathrm{l})$ at Mariout fish farm and the lowest $(92.72 \pm 38.32 \mu \mathrm{g} / \mathrm{l})$ recorded during summer at El-Abbassa fish farm (Table 2 and Fig. 2). There was a highly statistically significant difference $(\mathrm{P}<0.001)$ in iron concentration between water of all fish farms except between Bahr El-Baqar and Mariout fish farms (P > 0.05) (Table 3).

\section{2-Copper concentration:}

Results also showed that, the highest average of copper concentration in water was recorded at Bahr El-Baqar fish farm $(32.77 \pm 3.44 \mu \mathrm{g} / \mathrm{l})$ and the lowest value at El-Abbassa fish farm $(5.316 \pm 0.43 \mu \mathrm{g} / \mathrm{l})$. The maximum concentration of copper was recorded during spring $(35.99 \pm 3.01 \mu \mathrm{g} / \mathrm{l})$ at Bahr El-Baqar fish farm and the minimum value $(4.84 \pm 1.32 \mu \mathrm{g} / \mathrm{l})$ was record during summer at El-Abbassa fish farm (Table 2 and Fig. 2). There was a highly statistically significant difference $(\mathrm{P}<0.001)$ in average concentration of copper between water of all studied fish farms, except between Bahr El-Baqar and Mariout $(\mathrm{P}=0.0306)$ (Table 3). 


\section{3- Zinc concentration:}

As shown in (Table $2 \&$ Fig. 2), the highest average of zinc concentrations in water was recorded at Mariout fish farm $(73.72 \pm 5.79 \mu \mathrm{g} / \mathrm{l})$, and the lowest value $(22.81 \pm 10.32 \mu \mathrm{g} / \mathrm{l})$ at ElAbbassa fish farm. The highest concentration of zinc was recorded during spring $(79.12 \pm$ $10.44 \mu \mathrm{g} / \mathrm{l})$ at Mariout fish farm and the lowest concentration recorded during summer at ElAbbassa fish farm station $(14.10 \pm 2.18 \mu \mathrm{g} / \mathrm{l})$. There is a highly statistically significant difference $(\mathrm{P}<0.001)$ in average of zinc concentration between water of all studied fish farms (Table 3).

\section{4- Lead ion concentrations:}

Results showed that, the highest average of lead concentration in water was recorded at Bahr El-Baqar fish farm $(28.81 \pm 3.03 \mu \mathrm{g} / \mathrm{l})$ and the lowest value $(0.82 \pm 0.17 \mu \mathrm{g} / \mathrm{l})$ at El-Abbassa fish farm. The highest concentration of lead was recorded during spring $(33.09 \pm 9.96 \mu \mathrm{g} / \mathrm{l})$ at Bahr El-Baqar fish farm and the lowest $(0.64 \pm 0.25 \mu \mathrm{g} / \mathrm{l})$ recorded during summer at El-Abbassa fish farm (Table 2 and Fig. 2). There was a highly statistically significant difference $(\mathrm{P}<0.001)$ in lead concentration between water of all studied fish farms, except between Bahr El-Baqar and Mariout (P > 0.05) (Table 3).

Table (2): Heavy metal concentrations $(\mu \mathrm{g} / \mathrm{l})$ in water sample collected from different fish farms during the period of study (Data expressed as Mean \pm SD).

\begin{tabular}{|c|l|c|c|c|c|}
\hline \multirow{2}{*}{ Lakes } & Metals & \multicolumn{3}{|c|}{ Seasons } & \multirow{2}{*}{ Period Total } \\
\cline { 2 - 6 } & & Spring & Summer & Autumn & \\
\hline \multirow{3}{*}{$\begin{array}{c}\text { El- } \\
\text { Abbassa }\end{array}$} & Iron & $130.34 \pm 70.96$ & $126.71 \pm 23.03$ & $92.72 \pm 38.32$ & $116.59 \pm 16.94$ \\
\cline { 2 - 6 } & Copper & $5.89 \pm 0.96$ & $4.84 \pm 1.32$ & $5.22 \pm 0.69$ & $5.316 \pm 0.43$ \\
\cline { 2 - 6 } & Zinc & $37.32 \pm 14.50$ & $14.10 \pm 2.18$ & $17.01 \pm 3.35$ & $22.81 \pm 10.32$ \\
\cline { 2 - 6 } & Lead & $1.06 \pm 0.63$ & $0.64 \pm 0.25$ & $0.84 \pm 0.63$ & $0.823 \pm 0.17$ \\
\hline \multirow{4}{*}{ Edko } & Iron & $451.33 \pm 128.61$ & $279.51 \pm 58.98$ & $440.94 \pm 81.49$ & $390.59 \pm 78.66$ \\
\cline { 2 - 6 } & Copper & $17.04 \pm 4.74$ & $11.57 \pm 1.59$ & $14.72 \pm 3.46$ & $14.44 \pm 2.24$ \\
\cline { 2 - 6 } & Zinc & $23.80 \pm 7.02$ & $43.70 \pm 5.08$ & $27.29 \pm 3.48$ & $31.59 \pm 8.67$ \\
\cline { 2 - 6 } Mariout & Lead & $13.72 \pm 5.81$ & $27.96 \pm 1.96$ & $17.20 \pm 9.45$ & $19.62 \pm 6.06$ \\
\hline & Iron & $1043.94 \pm 58.83$ & $567.18 \pm 100.94$ & $917.02 \pm 80.97$ & $842.71 \pm 201.6$ \\
\cline { 2 - 6 } & Copper & $33.76 \pm 15.04$ & $29.00 \pm 1.85$ & $31.01 \pm 2.16$ & $31.25 \pm 1.95$ \\
\cline { 2 - 6 } & Zinc & $79.12 \pm 10.44$ & $65.68 \pm 6.84$ & $76.38 \pm 7.95$ & $73.72 \pm 5.79$ \\
\hline \multirow{3}{*}{$\begin{array}{c}\text { Bahr El- } \\
\text { Baqar }\end{array}$} & Lead & $27.29 \pm 5.34$ & $28.40 \pm 3.54$ & $27.73 \pm 8.73$ & $27.80 \pm 0.45$ \\
\cline { 2 - 6 } & Iron & $921.74 \pm 62.62$ & $898.60 \pm 60.53$ & $769.30 \pm 71.56$ & $863.21 \pm 67.07$ \\
\cline { 2 - 6 } & Zinc & $35.99 \pm 3.01$ & $27.99 \pm 1.77$ & $34.33 \pm 4.75$ & $32.77 \pm 3.44$ \\
\cline { 2 - 6 } & Lead & $33.09 \pm 9.96$ & $27.04 \pm 4.03$ & $26.32 \pm 3.02$ & $28.81 \pm 3.03$ \\
\hline
\end{tabular}

Table (3): Statistical analysis between different fish farms for different heavy metal concentrations in water samples

\begin{tabular}{|c|c|c|c|c|c|c|c|c|c|c|c|c|c|c|c|c|}
\hline \multirow{2}{*}{ Stations } & \multicolumn{4}{|c|}{ El-Abbassa } & \multicolumn{4}{|c|}{ Edko } & \multicolumn{4}{|c|}{ Mariout } & \multicolumn{4}{|c|}{ Bahr El-Baqar } \\
\hline & $\mathbf{F e}$ & $\mathrm{Cu}$ & $\mathbf{Z n}$ & $\mathbf{P b}$ & $\mathrm{Fe}$ & $\mathrm{Cu}$ & $\mathbf{Z n}$ & $\mathbf{P b}$ & $\mathbf{F e}$ & $\mathbf{C u}$ & $\mathbf{Z n}$ & $\mathbf{P b}$ & $\mathrm{Fe}$ & $\mathbf{C u}$ & $\mathbf{Z n}$ & $\mathbf{P b}$ \\
\hline El-Abbassa & & & & & $* *$ & $* *$ & $* *$ & $* *$ & $* *$ & $* *$ & $* *$ & $* *$ & $* *$ & $* *$ & $* *$ & $* *$ \\
\hline Edko & $* *$ & $* *$ & $* *$ & $* *$ & & & & & $* *$ & $* *$ & $* *$ & $* *$ & $* *$ & $* *$ & $* *$ & $* *$ \\
\hline Mariout & $* *$ & $* *$ & $* *$ & $* *$ & $* *$ & $* *$ & $* *$ & $* *$ & & & & & NS & NS & $* *$ & NS \\
\hline $\begin{array}{l}\text { Bahr El- } \\
\text { Baqar }\end{array}$ & $* *$ & $* *$ & $* *$ & $* *$ & $* *$ & $* *$ & $* *$ & $* *$ & NS & NS & $* *$ & NS & & & & \\
\hline
\end{tabular}

**: highly significant $(P<0.01)$; * significant $(P<0.05)$ and NS: non-significant $(P>0.05)$ 

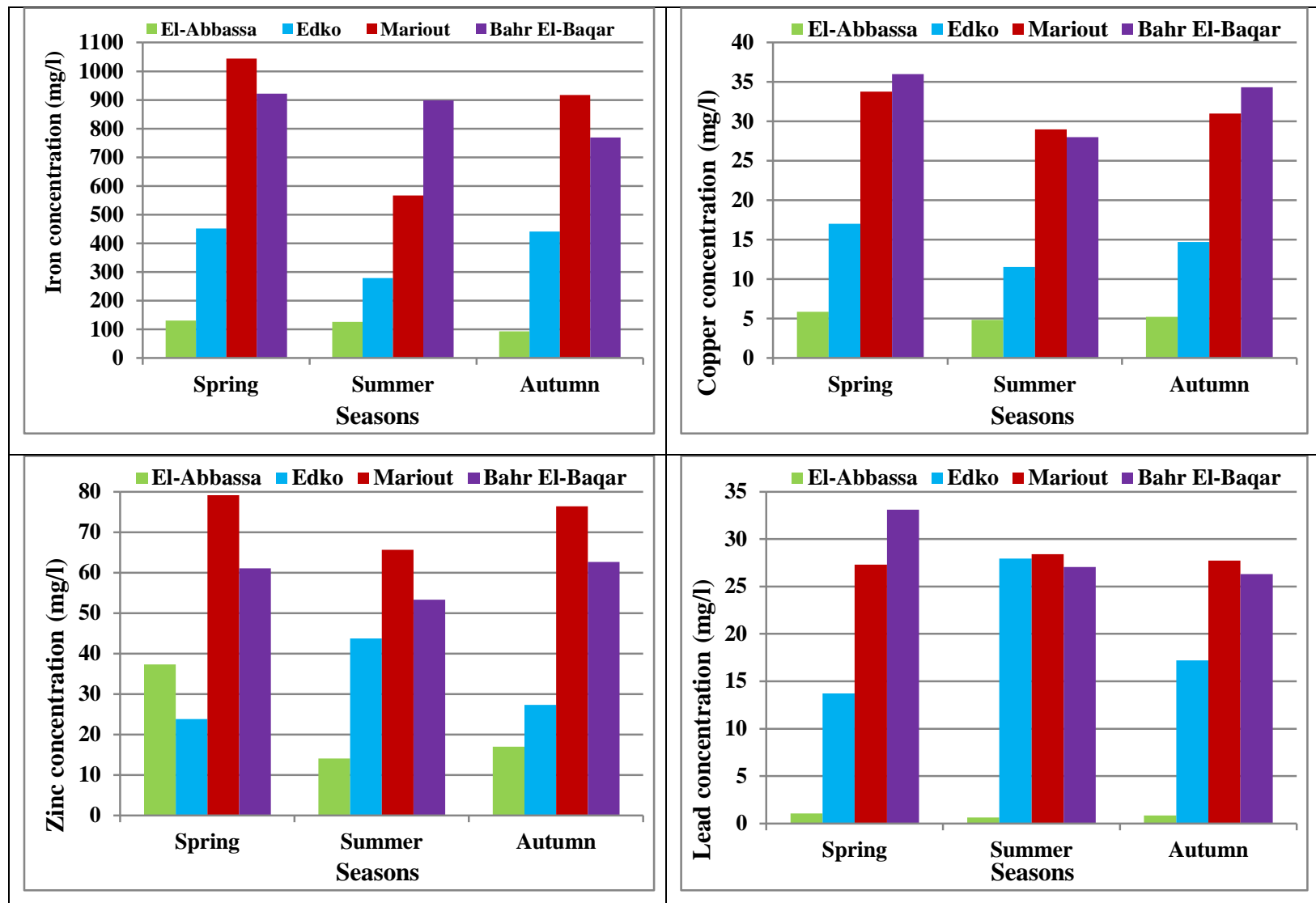

Figure (2): Heavy metals (iron, copper, zinc and lead) concentrations $(\mu \mathrm{g} / \mathrm{l})$ in water sample collected from different fish farms during the period of study.

\section{2- Heavy metals concentration $(\mathrm{mg} / \mathrm{kg}$ wet $\mathrm{wt})$ in sediment:}

\section{1- Iron ion concentrations:}

Results showed that the highest average value of iron concentration in sediment was recorded at Mariout fish farm $(42700.92 \pm 1923.79 \mathrm{mg} / \mathrm{kg})$ and the lowest value at El-Abbassa fish farm $(11809.39 \pm 433.32 \mathrm{mg} / \mathrm{kg})$. The highest concentration of iron was recorded during summer $(45325.61 \pm 782.15 \mathrm{mg} / \mathrm{kg})$ at Mariout fish farm and the lowest $(11443 \pm 746.17 \mathrm{mg} / \mathrm{kg})$ recorded during summer at El-Abbassa fish farm (Table 4 and Fig. 3). There was a highly statistically significant difference $(\mathrm{P}<0.001)$ between all sediment samples in all studied fish farms in iron concentration (Table 5).

\section{2-Copper ion concentrations:}

Result shows that, the highest concentration of copper concentration in sediment was recorded at Bahr El-Baqar fish farm $(327.69 \pm 11.90 \mathrm{mg} / \mathrm{kg})$ and the lowest value (57.54 \pm $6.01 \mathrm{mg} / \mathrm{kg}$ ) at El-Abbassa fish farm (Table 4 and Fig. 3). The highest copper concentration was recorded during spring season $(343.87 \pm 21.50 \mathrm{mg} / \mathrm{kg})$ at Bahr El-Baqar fish farm and the lowest was recorded during summer at El-Abbassa fish farm $(49.26 \pm 3.52 \mathrm{mg} / \mathrm{kg})$. There was a highly statistically significant difference $(\mathrm{P}<0.001)$ between all fish farms in their sediment copper content, except between Edko and Abbassa fish farms $(\mathrm{P}>0.05)$ (Table 5). 


\section{3-Zinc ion concentrations:}

As shown in (Table 4 \& Fig. 3), the highest average of zinc concentrations in sediment was recorded at Bahr El-Baqar fish farm $(343.16 \pm 19.98 \mathrm{mg} / \mathrm{kg})$, and the lowest value $(70.99 \pm$ $15.91 \mathrm{mg} / \mathrm{kg}$ ) at El-Abbassa fish farm. The maximum concentration of zinc was recorded during spring $(370.11 \pm 14.72 \mathrm{mg} / \mathrm{kg})$ at Bahr El-Baqar fish farm and the minimum value $(59.25 \pm 9.42$ $\mathrm{mg} / \mathrm{kg}$ ) was record during summer at El-Abbassa fish farm. There is a highly statistically significant difference $(\mathrm{P}<0.001)$ in average of zinc concentration between sediment of all fish farms, except between Bahr El-Baqar and Mariout fish farm stations ( $\mathrm{P}>0.05)$ (Table 5).

\section{4-Lead ion concentrations:}

Results showed that, the highest average of lead concentration in sediment was recorded at Bahr El-Baqar fish farm $(114.16 \pm 13.58 \mathrm{mg} / \mathrm{kg})$ and the lowest value $(1.18 \pm 0.17 \mathrm{mg} / \mathrm{kg})$ at El-Abbassa fish farm. The maximum lead concentration was recorded during spring (124.89 \pm $3.44 \mathrm{mg} / \mathrm{kg})$ at Bahr El-Baqar fish farm and the lowest $(0.93 \pm 0.56 \mathrm{mg} / \mathrm{kg})$ recorded during summer at El-Abbassa fish farm (Table 4 and Fig. 3). There was a highly significant difference $(\mathrm{P}<0.001)$ in lead concentration between water of all studied fish farms, except between Mariout and Edko fish farms $(\mathrm{P}>0.05)$ (Table 5).

Table (4): Heavy metal concentrations $(\mathrm{mg} / \mathrm{kg})$ recorded in sediment samples collected from different fish farms during the period of study (Data expressed as Mean \pm SD).

\begin{tabular}{|c|l|c|c|c|c|}
\hline \multirow{2}{*}{ Fish farm } & \multirow{2}{*}{ Metals } & \multicolumn{3}{|c|}{ Seasons } & \multirow{2}{*}{ Period Total } \\
\cline { 2 - 6 } & & Spring & Summer & Autumn & \\
\hline \multirow{3}{*}{ El-Abbassa } & Iron & $12418 \pm 1127.5$ & $11443 \pm 746.17$ & $11567 \pm 585.8$ & $11809 \pm 433.3$ \\
\cline { 2 - 6 } & Copper & $63.29 \pm 12.52$ & $49.26 \pm 3.52$ & $60.07 \pm 3.90$ & $57.54 \pm 6.01$ \\
\cline { 2 - 6 } & Zinc & $93.5 \pm 13.11$ & $59.25 \pm 9.42$ & $60.23 \pm 14.31$ & $70.99 \pm 15.91$ \\
\cline { 2 - 6 } & Lead & $1.31 \pm 0.96$ & $0.93 \pm 0.56$ & $1.30 \pm 0.27$ & $1.18 \pm 0.17$ \\
\hline \multirow{3}{*}{ Edko } & Iron & $28022 \pm 3359.6$ & $24970 \pm 3549.0$ & $26498 \pm 2962.4$ & $26497 \pm 1246.0$ \\
\cline { 2 - 6 } & Copper & $75.04 \pm 12.30$ & $66.09 \pm 17.28$ & $54.78 \pm 6.19$ & $65.31 \pm 8.28$ \\
\cline { 2 - 6 } & Zinc & $193.29 \pm 5.09$ & $181.10 \pm 20.67$ & $142.31 \pm 10.30$ & $172.23 \pm 21.73$ \\
\cline { 2 - 6 } Mariout & Lead & $21.82 \pm 1.84$ & $29.71 \pm 5.17$ & $25.48 \pm 1.02$ & $25.67 \pm 3.22$ \\
\hline & Iron & $42009 \pm 889.7$ & $45326 \pm 782.2$ & $40768 \pm 879.2$ & $42701 \pm 1923.8$ \\
\cline { 2 - 6 } & Copper & $132.41 \pm 3.89$ & $95.76 \pm 13.22$ & $121.88 \pm 41.42$ & $116.68 \pm 15.40$ \\
\cline { 2 - 6 } & Zinc & $333.34 \pm 22.45$ & $322.57 \pm 24.55$ & $299.95 \pm 43.79$ & $318.62 \pm 13.91$ \\
\cline { 2 - 6 } Bahr El- & Lead & $33.63 \pm 4.96$ & $29.33 \pm 5.87$ & $29.80 \pm 1.35$ & $30.92 \pm 1.92$ \\
\hline Baqar & Iron & $38722 \pm 2885.7$ & $35384 \pm 2284.5$ & $37264 \pm 1219.9$ & $37124 \pm 1366.5$ \\
\cline { 2 - 6 } & Copper & $343.87 \pm 21.50$ & $323.63 \pm 40.47$ & $315.57 \pm 51.78$ & $327.69 \pm 11.90$ \\
\cline { 2 - 6 } & Zinc & $370.11 \pm 14.72$ & $322.32 \pm 50.84$ & $337.06 \pm 19.99$ & $343.16 \pm 19.98$ \\
\cline { 2 - 6 } & Lead & $124.89 \pm 3.44$ & $122.61 \pm 35.98$ & $94.99 \pm 17.38$ & $114.16 \pm 13.58$ \\
\hline
\end{tabular}

Table (5): Statistical analysis between different fish farms for different heavy metal concentrations in sediment samples.

\begin{tabular}{|c|c|c|c|c|c|c|c|c|c|c|c|c|c|c|c|c|}
\hline \multirow{2}{*}{ Stations } & \multicolumn{4}{|c|}{ El-Abbassa } & \multicolumn{4}{|c|}{ Edko } & \multicolumn{4}{|c|}{ Mariout } & \multicolumn{4}{|c|}{ Bahr El-Baqar } \\
\hline & $\mathrm{Fe}$ & $\mathbf{C u}$ & $\mathbf{Z n}$ & $\mathbf{P b}$ & $\mathbf{F e}$ & $\mathrm{Cu}$ & $\mathbf{Z n}$ & $\mathbf{P b}$ & $\mathbf{F e}$ & $\mathbf{C u}$ & $\mathbf{Z n}$ & $\mathbf{P b}$ & $\mathbf{F e}$ & $\mathbf{C u}$ & $\mathbf{Z n}$ & $\mathbf{P b}$ \\
\hline El-Abbassa & & & & & $* *$ & NS & $* *$ & $* *$ & $* *$ & $* *$ & $* *$ & $* *$ & $* *$ & $* *$ & $* *$ & $* *$ \\
\hline Edko & $* *$ & NS & $* *$ & $* *$ & & & & & $* *$ & $* *$ & $* *$ & NS & $* *$ & $* *$ & NS & $* *$ \\
\hline Mariout & $* *$ & $* *$ & $* *$ & $* *$ & $* *$ & $* *$ & $* *$ & NS & & & & & $* *$ & $* *$ & $* *$ & $* *$ \\
\hline Bahr El-Baqar & $* *$ & $* *$ & $* *$ & $* *$ & $* *$ & $* *$ & $* *$ & $* *$ & $* *$ & $* *$ & NS & $* *$ & & & & \\
\hline
\end{tabular}

**: highly significant $(P<0.01)$; *: significant $(P<0.05)$ and NS: non-significant $(P>0.05)$ 

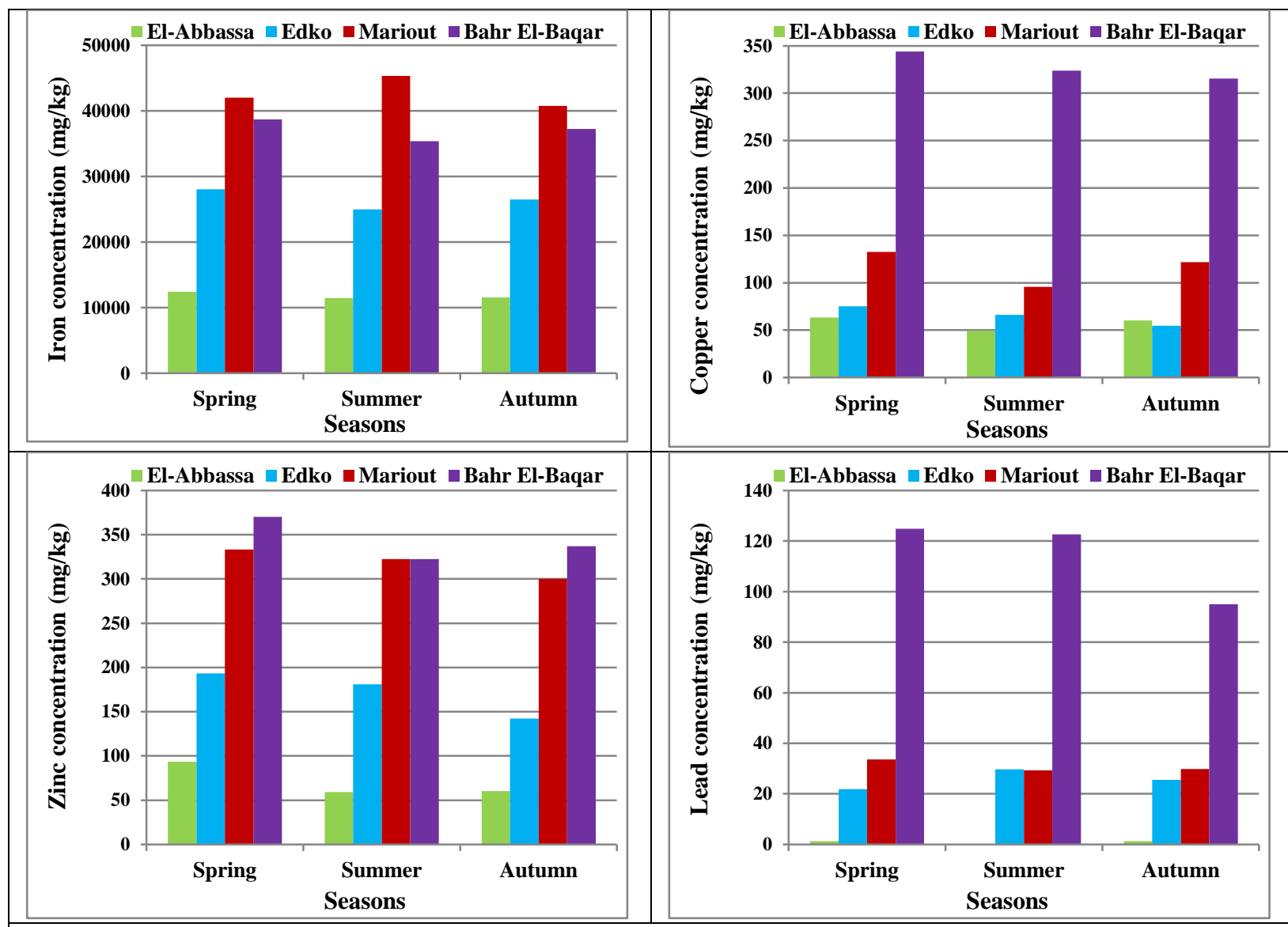

Figure (3): Heavy metals (iron, copper, zinc and lead) concentrations $(\mathrm{mg} / \mathrm{kg}$ ) in sediment sample collected from different fish farms during the period of study.

\section{3- Heavy metals concentration ( $\mathrm{mg} / \mathrm{kg}$ wet $\mathrm{wt}$ ) in fish tissues:}

\section{1- Iron ion concentrations:}

Results showed that the highest average value of iron concentration in muscles of Oreochromis niloticus was recorded at Bahr El-Baqar fish farm $(300.5 \pm 121.15 \mathrm{mg} / \mathrm{kg})$ and the lowest value at El-Abbassa fish farm $(81.46 \pm 33.98 \mathrm{mg} / \mathrm{kg})$. The highest concentration of iron was recorded during autumn $(454.77 \pm 85.79 \mathrm{mg} / \mathrm{kg})$ at Bahr El-Baqar fish farm and the lowest $(40.82 \pm 3.6 \mathrm{mg} / \mathrm{kg})$ recorded during spring at El-Abbassa fish farm (Table 6 and Fig. 4). There was a highly statistically significant difference $(\mathrm{P}<0.001)$ between all fish tissue samples from all studied fish farms in iron content, except between Edko and El-Abbassa fish farms $(\mathrm{P}<0.05)$ (Table 7).

\section{2-Copper ion concentrations:}

Result shows that, the highest concentration of copper concentration in fish tissue was recorded at Bahr El-Baqar fish farm $(31.62 \pm 9.02 \mathrm{mg} / \mathrm{kg})$ and the lowest value $(13.32 \pm 5.29$ $\mathrm{mg} / \mathrm{kg}$ ) at El-Abbassa fish farm station (Table 6 and Fig. 4). The highest copper concentration was recorded during autumn $(42.24 \pm 2.46 \mathrm{mg} / \mathrm{kg})$ at Bahr El-Baqar fish farm station and the lowest was recorded during spring at El-Abbassa fish farm station $(6.2 \pm 1.74 \mathrm{mg} / \mathrm{kg})$. There was a highly statistically significant difference $(\mathrm{P}<0.001)$ between all fish farms in their fish tissue copper content, except between Edko and Mariout fish farms ( $>0.05)$ (Table 7). 


\section{3-Zinc ion concentrations:}

As shown in (Table 6 \& Fig. 4), the highest average of zinc concentrations in sediment was recorded at Bahr El-Baqar fish farm $(134.26 \pm 46.67 \mathrm{mg} / \mathrm{kg})$, and the lowest value (46.16 \pm $13.87 \mathrm{mg} / \mathrm{kg}$ ) at El-Abbassa fish farm. The maximum concentration of zinc was recorded during summer $(175.01 \pm 16.21 \mathrm{mg} / \mathrm{kg})$ at Bahr El-Baqar fish farm and the minimum value $(23.26 \pm 1.83$ $\mathrm{mg} / \mathrm{kg}$ ) was record during spring at Edko fish farm. There is a highly significant difference (P $<0.001$ ) between all studied fish farms in their fish tissue zinc content, except between Edko and El-Abbassa fish farms ( $\mathrm{P}>0.05)$ (Table 7).

\section{4-Lead ion concentrations:}

Results showed that, the highest average of lead concentration in sediment was recorded at Bahr El-Baqar fish farm $(5.03 \pm 2 \mathrm{mg} / \mathrm{kg})$ and the lowest value $(0.68 \pm 0.61 \mathrm{mg} / \mathrm{kg})$ at ElAbbassa fish farm. The maximum lead concentration was recorded during autumn $(7.21 \pm 1.44$ $\mathrm{mg} / \mathrm{kg})$ at Bahr El-Baqar fish farm and the lowest $(0.02 \pm 0.01 \mathrm{mg} / \mathrm{kg})$ recorded during spring at El-Abbassa fish farm (Table 6, and Fig. 4). There was a highly statistically significant difference $(\mathrm{P}<0.001)$ between all studied fish farms in their fish tissue lead content, except between Edko and El-Abbassa fish farms ( $\mathrm{P}>0.05)$ (Table 7).

Table (6): Heavy metal concentrations ( $\mathrm{mg} / \mathrm{kg}$ ) recorded in fish tissue of $O$. niloticus collected from different fish farms during the period of study (Data expressed as Mean \pm SD).

\begin{tabular}{|c|l|c|c|c|c|}
\hline \multirow{2}{*}{ Lakes } & \multirow{2}{*}{ Metals } & \multicolumn{3}{|c|}{ Seasons } & \multirow{2}{*}{ Total } \\
\cline { 3 - 6 } & & Spring & Summer & Autumn & \\
\hline \multirow{4}{*}{ El-Abbassa } & Iron & $40.82 \pm 3.6$ & $79.57 \pm 9.23$ & $124 \pm 25.25$ & $81.46 \pm 33.98$ \\
\cline { 2 - 6 } & Copper & $6.2 \pm 1.74$ & $18.87 \pm 6.71$ & $14.9 \pm 1.8$ & $13.32 \pm 5.29$ \\
\cline { 2 - 6 } & Zinc & $26.86 \pm 2.44$ & $52.8 \pm 10.36$ & $58.83 \pm 12.56$ & $46.16 \pm 13.87$ \\
\cline { 2 - 6 } & Lead & $0.02 \pm 0.01$ & $0.53 \pm 0.09$ & $1.49 \pm 0.1$ & $0.68 \pm 0.61$ \\
\hline \multirow{4}{*}{ Edko } & Iron & $74.9 \pm 7.21$ & $138.06 \pm 21.79$ & $223.45 \pm 28.1$ & $145.47 \pm 60.87$ \\
\cline { 2 - 6 } & Copper & $8.98 \pm 0.59$ & $17.01 \pm 2.5$ & $22.37 \pm 6.93$ & $16.12 \pm 5.5$ \\
\cline { 2 - 6 } & Zinc & $23.26 \pm 1.83$ & $77.02 \pm 17.84$ & $110.42 \pm 38.37$ & $70.23 \pm 35.91$ \\
\cline { 2 - 6 } & Lead & $0.97 \pm 0.34$ & $2.78 \pm 0.68$ & $2.82 \pm 0.22$ & $2.19 \pm 0.86$ \\
\hline \multirow{3}{*}{$\begin{array}{c}\text { Bahr Elout } \\
\text { Baqar }\end{array}$} & Iron & $85.96 \pm 7.87$ & $212.53 \pm 39.03$ & $294.68 \pm 41.57$ & $197.72 \pm 85.85$ \\
\cline { 2 - 6 } & Copper & $13.99 \pm 3.03$ & $26.53 \pm 6.03$ & $38.9 \pm 3.4$ & $26.47 \pm 10.17$ \\
\cline { 2 - 6 } & Zinc & $73.42 \pm 10.25$ & $140.02 \pm 14.54$ & $160.38 \pm 21.28$ & $124.61 \pm 37.14$ \\
\cline { 2 - 6 } & Lead & $2.33 \pm 0.96$ & $3 \pm 0.56$ & $6.8 \pm 1.09$ & $4.04 \pm 1.97$ \\
\cline { 2 - 6 } & Copper & $158.82 \pm 6.97$ & $287.96 \pm 29.1$ & $454.77 \pm 85.79$ & $300.5 \pm 121.15$ \\
\cline { 2 - 6 } & Zinc & $20.19 \pm 1.24$ & $32.42 \pm 3.6$ & $42.24 \pm 2.46$ & $31.62 \pm 9.02$ \\
\cline { 2 - 6 } & Lead & $2.39 \pm 0.78$ & $5.5 \pm 1.01$ & $7.21 \pm 1.44$ & $5.03 \pm 2$ \\
\hline
\end{tabular}

Table (7): Statistical analysis between different fish farms for different heavy metal concentrations in fish tissues samples.

\begin{tabular}{|c|c|c|c|c|c|c|c|c|c|c|c|c|c|c|c|c|}
\hline \multirow{2}{*}{ Stations } & \multicolumn{4}{|c|}{ El-Abbassa } & \multicolumn{4}{|c|}{ Edko } & \multicolumn{4}{|c|}{ Mariout } & \multicolumn{4}{|c|}{ Bahr El-Baqar } \\
\hline & $\mathbf{F e}$ & $\mathbf{C u}$ & $\mathbf{Z n}$ & $\mathbf{P b}$ & $\mathbf{F e}$ & $\mathbf{C u}$ & $\mathbf{Z n}$ & $\mathbf{P b}$ & $\mathbf{F e}$ & $\mathbf{C u}$ & $\mathbf{Z n}$ & $\mathbf{P b}$ & $\mathbf{F e}$ & $\mathbf{C u}$ & $\mathbf{Z n}$ & $\mathbf{P b}$ \\
\hline El-Abbassa & & & & & * & NS & NS & * & $* *$ & $* *$ & $* *$ & $* *$ & $* *$ & $* *$ & $* *$ & $* *$ \\
\hline Edko & $*$ & NS & NS & $*$ & & & & & $*$ & $*$ & $* *$ & $* *$ & $* *$ & $* *$ & $* *$ & $* *$ \\
\hline Mariout & $* *$ & $* *$ & $* *$ & $* *$ & $*$ & * & $* *$ & $* *$ & & & & & $* *$ & NS & NS & $*$ \\
\hline Bahr El-Baqar & $* *$ & $* *$ & $* *$ & $* *$ & $* *$ & $* *$ & $* *$ & $* *$ & $* *$ & NS & NS & $*$ & & & & \\
\hline
\end{tabular}

**: highly significant $(\mathrm{P}<0.01)$; *: significant $(\mathrm{P}<0.05)$ and NS: non-significant $(\mathrm{P}>0.05)$ 


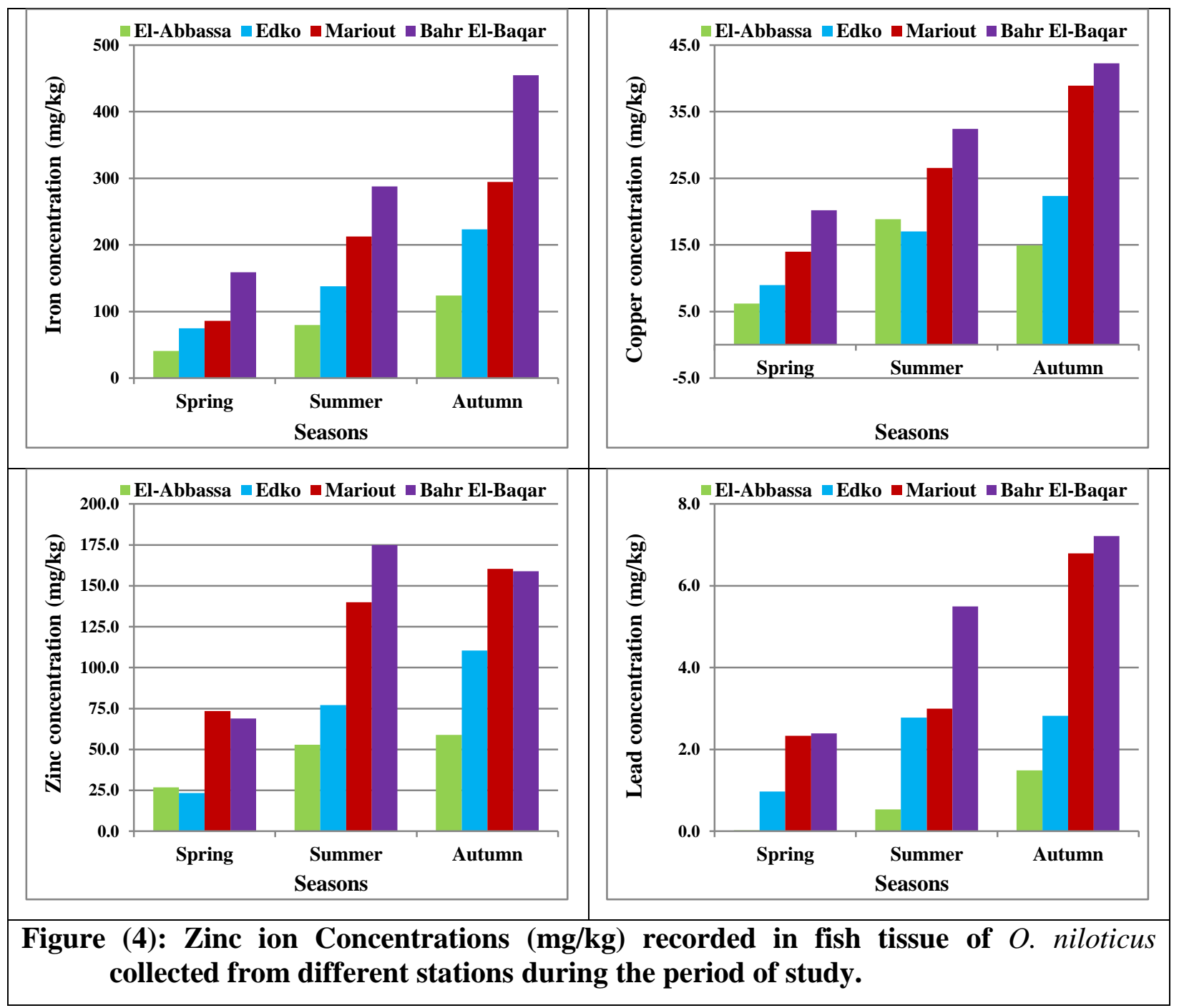

\section{Heavy metals pollution factors and indices:}

\section{1-Bio-Accumulation Factors (BAF):}

As shown in Table (8) the values of bio-accumulation factor of studied heavy metals (Fe, $\mathrm{Cu}, \mathrm{Zn}$, and $\mathrm{Pb}$ ) in muscle tissues of Nile tilapia, $O$. niloticus clarified that, the highest value of BAF (2641) was recorded for zinc from El-Abbassa fish farm, while the lowest value (111.3) was figured for lead which collected from Edko fish farm. The previous results confirmed that, the rank order of $\mathrm{BAF}$ for fish tissues was $\mathrm{Zn}>\mathrm{Cu}>\mathrm{Fe}>\mathrm{Pb}$. The rank order of BAF for fish farms was El-Abbassa > Edko > Bahr El-Baqa $r>$ Mariout.

\section{2-Bio-Sedimentation Factor (BSF):}

As shown in Table (8), the values of bio-sedimentation factor of heavy metals ( $\mathrm{Fe}, \mathrm{Cu}$, $\mathrm{Zn}$, and $\mathrm{Pb}$ ) for fish tissues showed that the maximum value of BSF (0.72) for zinc that accumulated in fish tissues collected from El-Abbassa fish farm and the lowest value (0.003) was recorded for iron, which accumulated in fish tissues collected from Mariout fish farm. The results confirmed that, the rank order of BSF for O. niloticus was $\mathrm{Zn}>\mathrm{Pb}>\mathrm{Cu}>\mathrm{Fe}$. While, the order of $\mathrm{BSF}$ for fish farms was ranked as El-Abbassa $>$ Edko $>$ Mariout $>$ Bahr El-Baqar. 


\section{3-Contamination Factor $\left(\mathrm{CF}_{\mathrm{w}}\right)$ for water:}

Results in Table (8) showed that, the highest value of $\mathbf{C F}_{\mathbf{W}}$ (7.95) was recorded for iron from Bahr El-Baqar fish farm, while the lowest value (0.37) was detected for lead which collected from El-Abbassa fish farm. The results confirmed that rank order of $\mathbf{C F}_{\mathbf{W}}$ for the tissue samples was $\mathrm{Pb}>\mathrm{Cu}>\mathrm{Zn}>\mathrm{Fe}$. While the order of $\mathbf{C F}_{\mathbf{W}}$ for fish farms was ranked as Bahr ElBaqar > Mariout $>$ Edko $>$ El-Abbassa.

\section{4-Contamination Factor $\left(\mathrm{CF}_{\mathrm{S}}\right)$ for sediment}

Results in Table (8) showed that, the highest value of $\mathbf{C F}_{\mathbf{S}}$ (7.28) was recorded for copper from Bahr El-Baqar fish farm, while the lowest value (0.06) was detected for lead from ElAbbassa fish farm. The rank order of $\mathbf{C F}_{\mathbf{S}}$ for O. niloticus was $\mathrm{Cu}>\mathrm{Zn}>\mathrm{Pb}>\mathrm{Fe}$. While the rank order of $\mathbf{C F}_{\mathbf{S}}$ for fish farms was Bahr El-Baqar $>$ Mariout $>$ Edko $>$ El-Abbassa.

\section{5-Pollution load Index (PLI) for water:}

Results in Table (8) showed that, the highest value of PLI (5.58) was recorded from Bahr El-Baqar fish farm, while the lowest value (1.40) was detected from El-Abbassa fish farm. The results confirmed that, the rank order of PLI for fish farms was Bahr El-Baqar > Mariout > Edko $>$ El-Abbassa.

\section{6- Pollution load Index (PLI) for sediment:}

Results in Table (8) showed that, the highest value of PLI (3.31) was recorded from Bahr El-Baqar fish farm, while lowest value (0.34) was recorded from El-Abbassa fish farm. The rank order of PLI for fish farms was Bahr El-Baqar > Mariout > Edko > El-Abbassa.

\section{7- Metal pollution index (MPI):}

Results in Table (8) clarified that, the highest value of MPI (50.1) was recorded from Bahr El-Baqar fish farm while the lowest value (12.6) was detected from El-Abbassa fish farm. The rank order of MPI for fish farm was Bahr El-Baqar > Mariout > Edko > El-Abbassa.

Table (8): Bio-Accumulation Factor (BAF), Bio-Sedimentation Factor (BSF), Contamination Factors (CF), Pollution load Index (PLI) and Metal Polution Index (MPI) of different heavy metals in Oreochromis noiloticus in studied fish farms during the period of study.

\begin{tabular}{|c|c|c|c|c|c|c|c|c|}
\hline \multirow{2}{*}{$\begin{array}{l}\text { Fish } \\
\text { farm }\end{array}$} & \multirow{2}{*}{ Metals } & \multicolumn{3}{|c|}{ Water } & \multicolumn{3}{|c|}{ Sediment } & \multirow{2}{*}{$\frac{\text { Fish tissues }}{\text { MPI }}$} \\
\hline & & BAF & $\mathrm{CF}$ & PLI & BSF & $\mathrm{CF}$ & PLI & \\
\hline \multirow{4}{*}{ 穴蒡 } & Iron & $759.5 \pm 524.58$ & $2.16 \pm 1.102$ & \multirow{4}{*}{ 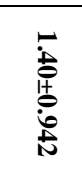 } & $0.01 \pm 0.006$ & $0.26 \pm 0.012$ & \multirow{4}{*}{ 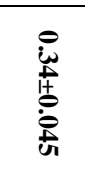 } & \multirow{4}{*}{ 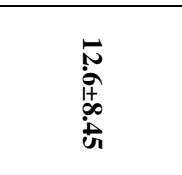 } \\
\hline & Copper & $2602.4 \pm 1440.08$ & $2.96 \pm 1.439$ & & $0.24 \pm 0.140$ & $1.28 \pm 0.167$ & & \\
\hline & Zinc & $2641.0 \pm 1670.07$ & $2.23 \pm 0.822$ & & $0.72 \pm 0.375$ & $0.74 \pm 0.199$ & & \\
\hline & Lead & $873.0 \pm 876.62$ & $0.37 \pm 0.405$ & & $0.58 \pm 0.560$ & $0.06 \pm 0.012$ & & \\
\hline \multirow{4}{*}{ 兽 } & Iron & $388.9 \pm 193.17$ & $3.85 \pm 1.973$ & \multirow{4}{*}{ 葛 } & $0.01 \pm 0.006$ & $0.58 \pm 0.035$ & \multirow{4}{*}{$\begin{array}{l}\dot{\vec{g}} \\
\stackrel{+1}{0} \\
\dot{8}\end{array}$} & \multirow{4}{*}{$\begin{array}{l}\stackrel{N}{+} \\
\stackrel{+}{+} \\
\stackrel{+}{+} \\
\dot{\omega}\end{array}$} \\
\hline & Copper & $1172.3 \pm 559.46$ & $3.58 \pm 1.496$ & & $0.26 \pm 0.145$ & $1.45 \pm 0.225$ & & \\
\hline & Zinc & $2262.1 \pm 1594.25$ & $3.4 \pm 2.126$ & & $0.44 \pm 0.330$ & $1.79 \pm 0.278$ & & \\
\hline & Lead & $111.3 \pm 47.75$ & $1.19 \pm 0.574$ & & $0.08 \pm 0.036$ & $1.28 \pm 0.200$ & & \\
\hline \multirow{4}{*}{ } & Iron & $259.5 \pm 155.70$ & $5.23 \pm 2.783$ & \multirow{4}{*}{ 离 } & $0.00 \pm 0.006$ & $0.93 \pm 0.053$ & \multirow{4}{*}{ 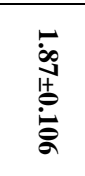 } & \multirow{4}{*}{$\begin{array}{l}+ \\
\stackrel{+}{\dot{\theta}} \\
\stackrel{+}{+} \\
\dot{0} \\
\dot{\infty}\end{array}$} \\
\hline & Copper & $861.2 \pm 422.51$ & $5.88 \pm 2.764$ & & $0.24 \pm 0.112$ & $2.59 \pm 0.417$ & & \\
\hline & Zinc & $1719.8 \pm 686.01$ & $6.03 \pm 2.199$ & & $0.39 \pm 0.158$ & $3.32 \pm 0.179$ & & \\
\hline & Lead & $145.4 \pm 86.89$ & $2.20 \pm 1.308$ & & $0.13 \pm 0.085$ & $1.55 \pm 0.116$ & & \\
\hline \multirow{4}{*}{ 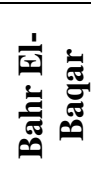 } & Iron & $361.3 \pm 212.39$ & $7.95 \pm 3.927$ & \multirow{4}{*}{ 峁 } & $0.01 \pm 0.006$ & $0.81 \pm 0.035$ & \multirow{4}{*}{ 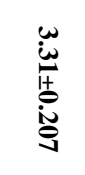 } & \multirow{4}{*}{ 崖 } \\
\hline & Copper & $983.3 \pm 367.44$ & $7.02 \pm 2.452$ & & $0.10 \pm 0.035$ & $7.28 \pm 0.324$ & & \\
\hline & Zinc & $2315.7 \pm 1093.30$ & $6.49 \pm 2.764$ & & $0.40 \pm 0.185$ & $3.58 \pm 0.257$ & & \\
\hline & Lead & $183.2 \pm 102.37$ & $2.74 \pm 1.328$ & & $0.05 \pm 0.031$ & $5.71 \pm 0.830$ & & \\
\hline
\end{tabular}




\section{DISCUSSION}

The present study revealed that, the highest concentrations of iron, copper, and lead in water were recorded at Bahr El-Baqar fish farm, and the highest value of zinc in water was recorded in Mariout fish farm. These results were much lower to that obtained by some authors (Saeed \& Shaker, 2008 at Manzalla; Abd El Azeem et al., 2012 at El-Abbassa; Mehanna, 2014 at Manzalla; Emara, 2015 at El-Abbassa and Bahr El-Baqar; Omran, 2016 at Bahr ElBaqar and Hamed, 2013 at Bahr El-Baqar). But these results were matched to that obtained by many others (Arafa, 2008 at Mariout; Saeed \& Shaker, 2008 at Edko and at Manzalla; Mehanna, 2014 in Manzalla; Zahran et al., 2015 at Bahr El-Baqar; Aly, 2016 at El-Abbassa; Omran, 2016 in Bahr El-Baqar; El-Batrawy, 2018 at Burullus and Ghannam, 2018 at ElAbbassa).

The present study revealed that, the highest concentrations of copper, zinc and lead in sediment were recorded at Bahr El-Baqar fish farm, and the highest value of iron in sediment was recorded in Mariout fish farm. These results were agreed that obtained by many authors (ElAmawy 2016; Shokr et al., 2016; Shalaby, 2017; Yun-Ru Ju et al., 2017; Khallaf et al., 2018).

Studies were focused on bioaccumulation of heavy metals in different parts of edible fish such as muscles (Yun-Ru Ju et al., 2017; El-Batrawy, 2018 and Azab et al., 2019). The present study showed that, the highest concentrations of iron, copper, zinc and lead in fish muscle tissues were recorded at Bahr El-Baqar fish farm. These higher concentrations were much lower to that obtained by some authors (Mehanna, 2014 in Manzalla; Shokr et al., 2016 at Bahr El-Baqar and Hamed, 2013 at Bahr El-Baqar). But these concentrations were matched to that obtained by many others (Saeed \& Shaker, 2008 at Edko; Abd-El-Khalek, 2012 at Mariout; Emara, 2015 at El-Abbassa; Zahran et al., 2015 at Bahr El-Baqar; Aly, 2016 at ElAbbassa; Omran, 2016 in Bahr El-Baqar; El-Batrawy, 2018 at Burullus and Ghannam, 2018 at El-Abbassa). But these concentrations were higher to that obtained by some authors (Abd El Azeem et al., 2012 and El-Batrawy, 2018).

Bio-accumulation of trace metals in fishes includes complex processes, which are controlled by exogenous and endogenous factors. Exogenous factors are reflected to environmental parameters such as metal bioavailability, temperature and alkalinity of ambient aquatic surroundings, whereas endogenous factors include species, age, size, physiological state and feeding behavior (Moiseenko \& Kudryavtseva, 2001). The present study indicated that, the highest averages of BAF were recoded to samples of El-Abbassa fish farm $(759.5 \pm 524.58$,

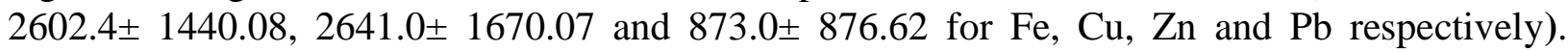
Results agree with El-Batrawy (2018) and Katip (2019), whom recorded the highest bioaccumulation factor for zinc, followed by copper, lead and iron. But these disagree with AbdelHamid et al. (2013) and Mortuza \& Al-Misned (2015).

A bio-sedimentation factor value greater than 1.00 indicates bioaccumulation existence (Barhoumi et al., 2009). Khallaf et al. (2018) mentioned that the accumulation of heavy metals in fish muscular tissue was found to be nearly comparable to the sediment. Thus, fish can be used as a surrogate for heavy metals pollution. Results of the present study revealed that, the maximal values of BSF $(0.72 \pm 0.375$ and $0.58 \pm 0.560)$ were obtained for zinc and lead, 
respectively in fish tissues collected from El-Abbassa fish farm. These results agree with AbdelHamid et al. (2013) and Mortuza \& Al-Misned (2015)

According to Harikumar \& Jisha (2010), results clarified that $\mathbf{C F}$ indicated very high contamination in Bahr El-Baqar fish farm, while El-Abbassa fish farm has a moderate contamination. These results were much higher than that obtained by El-Batrawy (2018) who obtained that $\mathrm{CF}$ values ranged between (1-1.9).

The degree of contamination from heavy metals could be evaluated by determining the contamination factor (CF), pollution load index (PLI), and metal pollution index (MPI) (Tomlinson et al., 1980 and Caeiro et al., 2005). The present results indicated that El-Abbassa fish farm has a low pollution and the other fish farms (Edko, Mariout and Bahr El-Baqar) have progressive deterioration of the site quality according to Caeiro et al. (2005).

\section{REFERENCES}

Abd El Azeem, M.H.; Hassan, A.M.; Hussein, M.M.; Abd El wahed, Z.H. and Saleh, R.E. (2012). Some heavy metal pollutants in fish farms and Effect of garlic on their bioaccumulation in Oreochromis niloticus. SCVMJ, XVII (1): 57-69.

Abdel-Hamid, A.M.; Gomaa, A.H. and El-Sayed, H.G.M. (2013). Studies on some heavy metals in the River Nile water and fish at Helwan area, Egypt. Egypt. J. Aquat. Biol. Fish., 17 (2): $105-126$.

Abd-El-Khalek, D.E.; El-Gohary, S.E. and El-Zokm, G.M. (2012). Assessment of heavy metals pollution in Oreochromis niloticus in EL-Max Fish Farm, Egypt. The Egyptian Journal of Experimental Biology (Zoology), 8(2): 215-222.

Abdel-Rasheed M.E. (2011). Ecological studies on Lake El-Manzalah with special reference to their water quality and sediment productivity. M.Sc. Thesis, Fac. Sci., Al-Azhar Uni, Cairo Egypt: 342 pp.

Abdel-Shafy, H.I. and Aly, R.O. (2002). Water issue in Egypt: Resources, pollution and protection endeavors. CEJOEM, 8(1): 3-21.

Afami, A. and Okorie, P.U. (2008). Some aspect of the reproductive biology of Tilapia mariae (Boulenger 1901) in a small lake in South Eastern Nigeria. Afri J. of Biotech., 7(14): 24782482.

Al Naggar, Y.; Khalil, M.S. and Ghorab, M.A. (2018). Environmental pollution by heavy metals in the aquatic ecosystems of Egypt. Toxicology, 3(1): 1-9.

Al-Haidarey, M.G.S. (2009). Assessment and Sources of some Heavy Metals in Mesopotamian Marshes. $\mathrm{PhD}$ thesis, Univ. of Baghdad, College of Science for Women,155pp.

Al-Kenawy, D.A. and Aly, N.A. (2015). Levels of some heavy metals in muscles and liver of freshwater farmed fish at abbassa. Abbassa Int. J. Aqua, 8(1), pp.20-35.

Aly, M.Y.M., (2016). Comparison of heavy metals levels in muscles, liver and gills of three fish species collected from agricultural drainage water at El-Abbassa fish farm. Egypt. J. Aquat. Biol. \& Fish., 20(3): 103- 112. 
Arafa, M.M. and Ali, A.T. (2008). Effect of some heavy metal's pollution in Lake Mariout on Oreochromis niloticus fish. Egypt. J. Comp. Path. \& Clinic. Path. 21(3):191- 201.

Azab, A. M.; Aly-Eldeen, M. A.; Khalaf-Allah, H. M. M. and El-Battal, M. M. A. (2019). Effect of heavy metals on the ovary of Tilapia zillii in some canals of Nile Delta area, Egypt. Egyptian Journal of Aquatic Biology \& Fisheries, 23(3): 329 -345.

Balasim, H.M. (2013). Assessment of some heavy metals' pollution in water, sediments and Barbus xanthopterus (Heckel, 1843) in Tigris River at Baghdad city, M. Sc. Thesis, Fac. Sci. Baghdad Uni., Iraq, 242 pp.

Barhoumi, S.; Messaoudi, I.; Deli, T.; Sa ïd, K. and Kerkeni, A. (2009). Cadmium bioaccumulation in three benthic fish species, Salaria basilisca, Zosterisessor ophiocephalus and Solea vulgaris collected from the Gulf of Gabes in Tunisia. J. Environ. Sci., 21: 980984.

Caeiro, S.; Costa, M.H. and Ramos, T.B. (2005). Assessing heavy metal contamination in sado estuary sediment: an index analysis approach. Ecol Ind 5:151-169.

David, K.E. and Janice, W.R. (1989). Acid digestion for sediments, sludges, soils, and solid wastes. A proposed alternative to EPA SW 846 Method 3050, Environ. Sci. Technol., 23: 898.

Duruibe, J.O.; Ogwuegbu, M.C. and Egwurugwu, J.N. (2007). Heavy metal pollution and human bio-toxic effects. International Journal of Physical Sciences, 2: 112-118.

ECDG, (2002). European Commission DG ENV. E3 Project ENV. E.3/ETU/0058. Heavy metals in waste.

El-Amawy, A.A.B. (2016). Effect of some pollutants on quality and water characters of the River Nile at Tanta and Kafr El-Zayat, Egypt. M.Sc. Thesis, Fac. Sci., Al-Azhar Univ. Egypt, Pp: 470.

El-Batrawy, O.A.; El-Gammal, M.I.; Mohamadein, L.I.; Darwish, D.H. and El-Moselhy, K.M. (2018). Impact assessment of some heavy metals on tilapia fish, Oreochromis niloticus, in Burullus Lake, Egypt. The Journal of Basic and Applied Zoology, 79(1), p.13.

El-Enany, H.R. (2004). Ecological and biological studies on Lake Manzalah with special reference to their water quality and sediment productivity. M. Sc.Thesis, Fac. Sci., Al-Azhar Univ.

El-Zokm, G.M.; Tadros, H.R.Z.; Okbah, M.A. and Ibrahim, G.H. (2018). Eutrophication assessment using TRIX and Carlson's indices in Lake Mariout Water, Egyptian Journal of Aquatic Biology \& Fisheries 22(5): 321- 339.

Emara, M.M.; Farag, R.S.; Dawah, A.A. and Fathi, M. (2015). Assessment of heavy metals concentration in water and edible tissues of Nile tilapia (Oreochromis niloticus) from two fish farms irrigated with different water sources, Egypt. International Journal of Environment, 4(1): 108-115.

Evans, D.W.; Dodoo, D.K. and Hanson, P.J. (1993). Trace elements concentrations in fish livers. Implications of variations with fish size in pollution monitoring. Mar. Pollut. Bull., 26:329-334.

FAO (1992). The state of food and agriculture. Food and Agriculture Organization of the United Nations, Rome, pp: 280.

Ghannam, E.H. and Aly, Y.M. (2018). Influence of different water resources on physical, chemical and heavy metals levels of El-Abbassa Fish Farms, El-Sharqia Governorate, Egypt. Egyptian Journal of Aquatic Biology and Fisheries, 22(5 (Special Issue): 165-174. 
Hakanson, L. (1980). An ecological risk index aquatic pollution control. A sedimentological approach. Water. Res. 14: 975-1001.

Hamed, Y.A.; Abdelmoneim, T.S.; ElKiki, M.H.; Hassan M.A. and Berndtsson R. (2013). Assessment of heavy metals pollution and microbial contamination in water, sediments and fish of Lake Manzala, Egypt, Life Science Journal, 10(1): 86-99.

Harikumar, P. S. \& Jisha, T.S. (2010). Distribution pattern of trace metal pollutants in the sediments of an urban wetlands in the southwest coast of India. International Journal of Engineering, 2(5): 840850.

Katip, A. (2019). Bioaccumulation of trace metals in wastewater-fed aquaculture: A case study in Turkey. Polish Journal of Environmental Studies, 28(6): 4221- 4238.

Khallaf, E.A.; Authman, M.M.N and Alnenaei, A.A. (2018). Contamination and ecological hazard assessment of heavy metals in freshwater sediments and Oreochromis niloticus (Linnaeus, 1758) fish muscles in a Nile River Canal in Egypt. Environ. Sci. Poll. Res., 25: 13796-13812.

MacFarlane, G.B. and Burchett, M.D. (2000). Cellular distribution of $\mathrm{Cu}, \mathrm{Pb}$ and $\mathrm{Zn}$ in the Grey Mangroove, Avicemmia marina (Forsk). Vierh Aquat. Bot., 68: 45 - 49.

Mansour, S.A. and Sidky, M.M. (2002). Ecotoxicological studies. 3. Heavy metals contaminating water and fish from Fayoum Governorate, Egypt. Food Chem.,78: 15-22.

Martin, T.D. and Martin, E.R. (1989). Evaluation of method 2002 sample preparation procedure for spectrochemical analyses of total recoverable elements, U.S. Environmental Protection Agency, office of research and development, environmental monitoring systems laboratory, Cincinnati, Ohio.

Mason, C.F. (2002). Biology of freshwater pollution. 4th ed. Essex Univ. England. 387 pp.

Mehanna, S.; Shaker, I.M. and Farouk, A.E.D. (2014). Impacts of excessive fishing effort and heavy metals pollution on the Tilapia production from Lake Manzalah. 4th Conference of Central Laboratory for Aquaculture Research (2014): 57-74.

Moiseenko, T.I. and Kudryavtseva, L.P. (2001). Trace metal accumulation and fish pathologies in areas affected by mining and metallurgical enterprises in the Kola Region, Russia. Environ. Pollut. 114: $285-297$.

Momtaz, M. (2002). Geochemical studies of Heavy Metals in the Seawater along Karachi Makran Coast, in Chemi, Karachi Univ., 414 pp.

Mortuza, G.M. and Al-Misned, F.A. (2015). Heavy metal concentration in two freshwater fishes from Wadi Hanifah (Riyadh, Saudi Arabia) and evaluation of possible health hazard to consumers. Pakistan. J. Zool., 47(3): 839 - 845.

Nussey, G. (1998). Metal Ecotoxicology of the Upper Olifants River at selected localities and the effect of Copper and Zinc on Fish Blood Physiology, Ph.D thesis, Rand Afrikaans Uni., SA, 412 pp.

Omran, E.E. (2016). Environmental modelling of heavy metals using pollution indices and multivariate techniques in the soils of Bahr El Baqar, Egypt. Model. Earth Syst. Environ. 2:119.

Orata, F. \& Birgen, F. (2016). Fish tissue bio-concentration and interspecies uptake of heavy metals from waste water lagoons. Journal of Pollution Effects \& Control., 4(2): 157 pp.

Saad S.A.; Magdy A.M.; Ranya, A.A. and Mohamed A.G. (2017). Assessment of the physico-chemical characteristics and water quality analysis of Mariout lake, Southern of Alexandria, Egypt. J. of Env. \& Analytical Toxicology, 7(1): 421-440. 
Saeed, S.M. \& Sakr, S.F. (2008). Impact of cage-fish culture in the river Nile on physico-chemical characteristics of water, metals accumulation, histological and some biochemical parameters in fish. Abbassa Int. J. Aqua., 1A: 179-202.

Saeed, S.M. \& Shaker, I.M. (2008). Assessment of heavy metals pollution in water and sediments and their effect on Oreochromis niloticus in the Northern Delta lakes, Egypt. $8^{\text {th }}$ International Symposium on Tilapia in Aquaculture, ISTA, Egypt, 475-490.

Shalaby, B.; Samy, Y.M.; Mashaly, A.O. and El Hefnawy, M.A.A. (2017). Comparative geochemical assessment of heavy metal pollutants among the Mediterranean Deltaic Lakes sediments (Edku, Burullus and Manzala), Egypt. Egyptian Journal of Chemistry, 60(3): 361-378.

Shokr, M.S.; El Baroudy, A.A.; Fullen, M.A.; El-beshbeshy, T.R.; Ramadan, A.R.; Abd El Halim, A. and Jorge, M.C.O. (2016). Spatial distribution of heavy metals in the middle Nile delta of Egypt. International Soil and Water Conservation Research, 4(4): 293-303.

Subasinghe, R.; Soto, D. and Jia, J. (2009). Aquaculture and its role in sustainable development. Rev. Aquacult., 1: 2- 9.

Tomlinson, D.C.; Wilson, D.J.; Harris, C.R. and Jeffrey, D.W. (1980). Problem in assessment of heavy metals in estuaries and the formation of pollution index. Helgol. Wiss. Meeresunlter, 33: 566575.

UNEP; FAO; IAEA and IOC (1984). Sampling of selected marine organisms and sample preparation for trace metal analysis. Reference Methods for Marine Pollution Studies No. 7, Rev. 2, UNEP, Nairobi.

Wang, Q.; Cheng, L.; Liu, J.; Li, Z.; Xie, S. and De Silva, S.S. (2015). Freshwater aquaculture in PR China: Trends and prospects. Rev. Aquacult., 7(4): 283-302.

Yun-Ru Ju, C.; C.W.; Chen, C.F.; Chuang, X.Y. and Dong, C.D. (2017). Assessment of heavy metals in aquaculture fishes collected from southwest coast of Taiwan and human consumption risk. International Biodeterioration \& Biodegradation, 124: 314- 325.

Zahran, M.A.E.K.; El-Amier, Y.A.; Elnaggar, A.A.; Mohamed, H.A.E.A. and El-Alfy, M.A.E.H. (2015). Assessment and distribution of heavy metals pollutants in Manzala Lake, Egypt. Journal of Geoscience and Environment Protection, 3(06): pp.107.

\section{الملخص العربي}

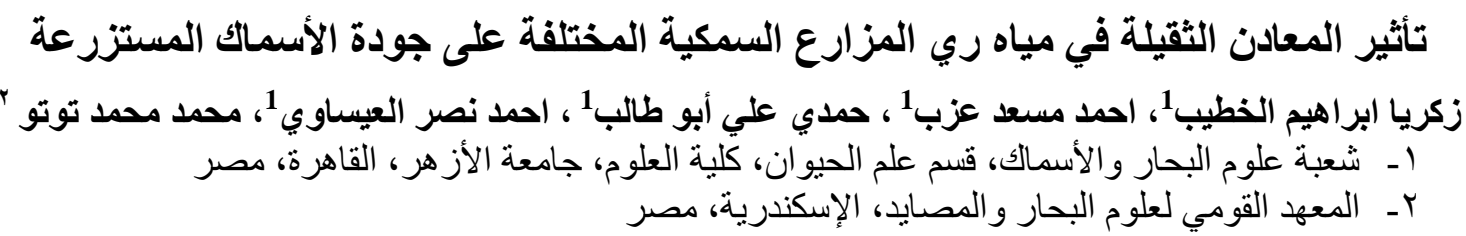

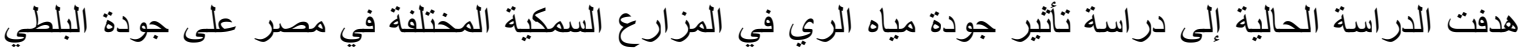

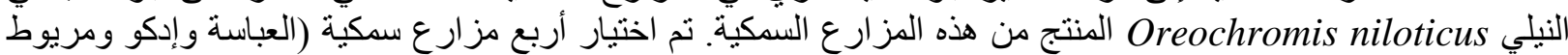

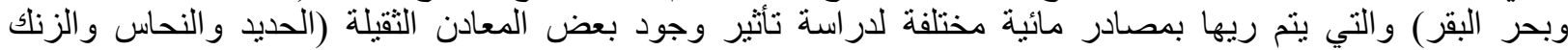

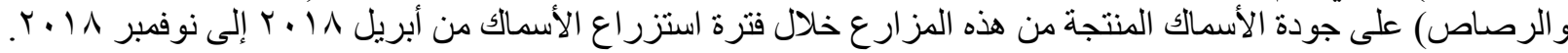
أوضحت النتائج أن معظم التركيزات المرتفعة للمعادن الثقبلة في الماءو والرواسب والأنسجة العضلية لأسماك البلطي

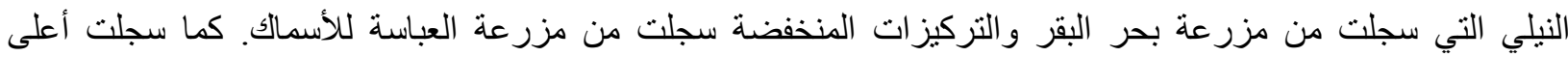

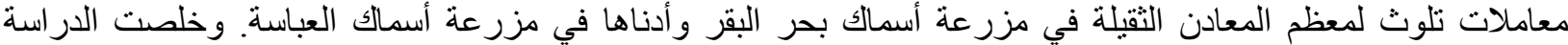

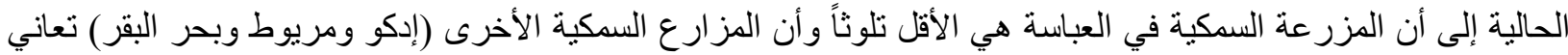
من تدهور تدريجي في جودة المياة والذي ينعكس على جودة الأسماك المنتجة. 